\title{
A homogenization approach for the motion of motor proteins
}

\author{
S. Mirrahimi and P. E. Souganidis
}

\begin{abstract}
We consider the asymptotic behavior of an evolving weakly coupled Fokker-Planck system of two equations set in a periodic environment. The magnitudes of the diffusion and the coupling are, respectively, proportional and inversely proportional to the size of the period. We prove that, as the period tends to zero, the solutions of the system either propagate (concentrate) with a fixed constant velocity (determined by the data) or do not move at all. The system arises in the modeling of motor proteins which can take two different states. Our result implies that, in the limit, the molecules either move along a filament with a fixed direction and constant speed or remain immobile.
\end{abstract}

Mathematics Subject Classification (2010). 35B25, 35B27, 49L25, 92C05.

Keywords: Hamilton-Jacobi equations, Homogenization, Molecular motor, Singular perturbation, Viscosity solutions.

\section{Introduction}

We study the asymptotics, as $\varepsilon \rightarrow 0$, of the weakly coupled Fokker-Planck system of two equations set

$$
\left\{\begin{array}{l}
n_{\varepsilon, t}^{1}-\varepsilon \Delta n_{\varepsilon}^{1}-\operatorname{div}_{x}\left(n_{\varepsilon}^{1} D_{y} \psi\left(\frac{x}{\varepsilon}\right)\right)+\frac{1}{\varepsilon} \nu^{1}\left(\frac{x}{\varepsilon}\right) n_{\varepsilon}^{1}=\frac{1}{\varepsilon} \nu^{2}\left(\frac{x}{\varepsilon}\right) n_{\varepsilon}^{2} \\
n_{\varepsilon, t}^{2}-\varepsilon \Delta n_{\varepsilon}^{2}+\frac{1}{\varepsilon} \nu^{2}\left(\frac{x}{\varepsilon}\right) n_{\varepsilon}^{2}=\frac{1}{\varepsilon} \nu^{1}\left(\frac{x}{\varepsilon}\right) n_{\varepsilon}^{1} .
\end{array} \quad \text { in } \mathbb{R}^{d} \times(0, \infty),\right.
$$

Systems like (1) have been used to model the motion of motor proteins along molecular filaments or microtubules $[2,9,13,14,20,21]$. The intracellular transport in eukaria is attributed to motor proteins that transform the chemical energy into mechanical motion. For example myosins, which are known for

P. E. Souganidis was partially supported by the National Science Foundation. 
their role in the muscle contraction, move along actin filaments and kynesins move along microtubules

In (1) the molecules have the conformations 1 and 2 with densities $n_{\varepsilon}^{1}$ and $n_{\varepsilon}^{1}$ respectively and are influenced by the periodic potential $\psi$ provided by the filaments (see [19]). The (periodic) functions $\nu^{1}$ and $\nu^{2}$ indicate the rates of change between the two states. Under some asymmetry assumptions on the potential, we prove that, as $\varepsilon$ goes to 0 , the proteins move along a fixed filament with a constant speed. The existence of traveling waves, the asymptotic speed of propagation for large time as well as the presence of concentration effects for models with diffusion and a periodic drift have been studied in many papers; for instance see $[8,10]$. A system similar to (1) also appears in the stochastic Stokes' drift where particles are suspended in a liquid and are subjected to diffusion and a net drift due to the presence of a wave in the liquid [7]. From the mathematical point of view, we refer to [1] for a related homogenization problem as well as to [15] for the homogenization of a similar equation with parabolic scaling. In particular, we observe some similarities in the results for our system with hyperbolic scaling and the case of a unique equation with parabolic scaling in the latter article (see Sect. 7).

To formulate our result for the densities $n_{\varepsilon}^{1}$ and $n_{\varepsilon}^{2}$ next we introduce the assumptions we will be using throughout the paper. In addition to

$\nu^{1}, \nu^{2}$ and $\psi$ are smooth and 1-periodic and $\nu^{1}>0, \nu^{2}>0$,

we assume that

$$
\begin{gathered}
n_{\varepsilon}^{1}(x, 0)>0 \text { and } n_{\varepsilon}^{2}(x, 0)>0 \text { for all } x \in \mathbb{R}^{d}, \\
I_{\varepsilon}^{0}=\int_{\mathbb{R}} n_{\varepsilon}^{1}(x, 0) d x+\int_{\mathbb{R}} n_{\varepsilon}^{2}(x, 0) d x \rightarrow I_{0}>0, \quad \text { as } \quad \varepsilon \rightarrow 0,
\end{gathered}
$$

$$
\left\{\begin{array}{l}
\lim _{\varepsilon \rightarrow 0} \varepsilon \ln n_{\varepsilon}^{1}(\cdot, 0)=\lim _{\varepsilon \rightarrow 0} \varepsilon \ln n_{\varepsilon}^{2}(\cdot, 0)=-\infty \text { locally uniformly in } \mathbb{R}^{d} \backslash\{0\}, \\
\limsup _{\substack{y \rightarrow 0 \\
\varepsilon \rightarrow 0}} \varepsilon \ln n_{\varepsilon}^{i}(y, 0) \leq 0 \text { for } i=1,2,
\end{array}\right.
$$

and, there exist constants $A>0$ and $B$ such that, for all $x \in \mathbb{R}^{d}$ and $i=1,2$,

$$
n_{\varepsilon}^{i}(x, 0) \leq e^{\varepsilon^{-1}(-A|x|+B)} .
$$

Let $\delta$ denote the Dirac mass at the origin. Our first result is

Theorem 1.1. Assume (2), (3), (4), (5) and (6). There exists $\bar{v} \in \mathbb{R}^{d}$ such that, as $\varepsilon \rightarrow 0$ and in the sense of measures,

$$
n_{\varepsilon}^{1}(t, x)+n_{\varepsilon}^{2}(t, x) \rightarrow \delta(x-t \bar{v}) I_{0} .
$$

To prove Theorem 1.1 we analyze the behavior, as $\varepsilon \rightarrow 0$, of the functions $R_{\varepsilon}^{1}, R_{\varepsilon}^{2}: \mathbb{R}^{d} \times[0, \infty) \rightarrow \mathbb{R}$ which are obtained from $n_{\varepsilon}^{1}$ and $n_{\varepsilon}^{2}$ by the classical exponential change of variable (we show later in the paper that $n_{\varepsilon}^{1}>0$ and $\left.n_{\varepsilon}^{2}>0\right)$

$$
n_{\varepsilon}^{1}=\exp \left(-R_{\varepsilon}^{1} / \varepsilon\right) \quad \text { and } \quad n_{\varepsilon}^{2}=\exp \left(-R_{\varepsilon}^{2} / \varepsilon\right)
$$


which is possible since, as we show in the next section, $n_{\varepsilon}^{1}>0$ and $n_{\varepsilon}^{2}>0$. and solve the system

$$
\begin{cases}R_{\varepsilon, t}^{1}-\varepsilon \Delta_{x} R_{\varepsilon}^{1}+\left|D_{x} R_{\varepsilon}^{1}\right|^{2}-D_{y} \psi\left(\frac{x}{\varepsilon}\right) \cdot D_{x} R_{\varepsilon}^{1}+\Delta_{y} \psi\left(\frac{x}{\varepsilon}\right) & \\ \quad+\nu^{2}\left(\frac{x}{\varepsilon}\right) \exp \left(\frac{R_{\varepsilon}^{1}-R_{\varepsilon}^{2}}{\varepsilon}\right)=\nu^{1}\left(\frac{x}{\varepsilon}\right) & \text { in } \mathbb{R}^{d} \times(0, \infty) \\ R_{\varepsilon, t}^{2}-\varepsilon \Delta_{x} R_{\varepsilon}^{2}+\left|D_{x} R_{\varepsilon}^{2}\right|^{2}+\nu^{1}\left(\frac{x}{\varepsilon}\right) \exp \left(\frac{R_{\varepsilon}^{2}-R_{\varepsilon}^{1}}{\varepsilon}\right)=\nu^{2}\left(\frac{x}{\varepsilon}\right), & \end{cases}
$$

where we use the subscript $x$ and $y$ to differentiate between differentiation with respect to $x$ and the fast variable $y=x / \varepsilon$.

To state the second main result we recall that the "half-relaxed" upper and lower limits, denoted $\bar{R}^{i}$ and $\underline{R}^{i}$ respectively, of the family $\left(R_{\varepsilon}^{i}\right)_{\varepsilon>0}$ are given, for $(x, t) \in \mathbb{R}^{d} \times[0, \infty)$ and $i=1,2$, by

$$
\underline{R}^{i}(x, t)=\liminf _{\substack{(y, s) \rightarrow(x, t) \\ \varepsilon \rightarrow 0}} R_{\varepsilon}^{1}(y, s) \text { and } \bar{R}^{i}(x, t)=\limsup _{\substack{(y, s) \rightarrow(x, t) \\ \varepsilon \rightarrow 0}} R_{\varepsilon}^{1}(y, s) .
$$

We have:

Theorem 1.2. Assume (2) and (3). Then

(i) $\bar{R}^{1}=\bar{R}^{2}$ and $\underline{R}^{1}=\underline{R}^{2}$ in $\mathbb{R}^{d} \times(0, \infty)$.

(ii) There exists a strictly convex $\bar{H} \in \mathrm{C}^{1}\left(\mathbb{R}^{d}\right)$ satisfying $\bar{H}(0)=0$ and, for some $C>0, \bar{H}(p) \geq|p|^{2}-C$ such that $\bar{R}=\bar{R}^{1}=\bar{R}^{2}$ and $\underline{R}=\underline{R}^{1}=\underline{R}^{2}$ are respectively subsolution and supersolution of

$$
\bar{R}_{t}+\bar{H}\left(\bar{R}_{x}\right) \leq 0 \quad \text { and } \quad \underline{R}_{t}+\bar{H}\left(\underline{R}_{x}\right) \geq 0 \quad \text { in } \mathbb{R}^{d} \times(0, \infty) .
$$

(iii) Assume, in addition, (5). Then

$$
\begin{gathered}
\bar{R}^{1}=\bar{R}^{2}=\underline{R}^{1}=\underline{R}^{2}=+\infty, \quad \text { in }\left(\mathbb{R}^{d} \backslash\{0\}\right) \times\{0\}, \\
\underline{R}^{1}(0,0) \geq 0 \text { and } \underline{R}^{2}(0,0) \geq 0 .
\end{gathered}
$$

(iv) The special direction $\bar{v}$ in Theorem 1.1 is

$$
\bar{v}=D \bar{H}(0) .
$$

The asymptotic behavior of a time-independent version of (1) set in $[0,1]$, which is also controlled by the same effective Hamiltonian $\bar{H}$, was studied in [27] where it was proved that, for asymmetric potentials, the mass concentrates at either $x=0$ or $x=1$. The asymmetry condition of [27] is $D \bar{H}(0) \neq 0$. This behavior is in agreement with our study of the time dependent problem. since we prove that the proteins move with a constant direction and spread along the filaments or the microtubules. Indeed we prove here that, if $\bar{v}=D \bar{H}(0) \neq 0$, the proteins (mass) move (spread) with constant velocity $\bar{v}$. Hence the mass concentrates, for large times, on one end point of the filament, if the latter is assumed to have finite length. We also refer to [25] for a large deviation approach for the asymptotic behavior of the stationary solution of a similar model but with two potentials and to [26] for the study of flashing ratchets. 
By slight modifications of the proofs, all the results in this paper extend to systems with two potentials like

$$
\left\{\begin{array}{l}
n_{\varepsilon, t}^{1}-\varepsilon \Delta n_{\varepsilon}^{1}-\operatorname{div}_{x}\left(n_{\varepsilon}^{1} D_{y} \psi^{1}\left(\frac{x}{\varepsilon}\right)\right)+\frac{1}{\varepsilon} \nu^{1}\left(\frac{x}{\varepsilon}\right) n_{\varepsilon}^{1}=\frac{1}{\varepsilon} \nu^{2}\left(\frac{x}{\varepsilon}\right) n_{\varepsilon}^{2} \\
n_{\varepsilon, t}^{2}-\varepsilon \Delta n_{\varepsilon}^{2}-\operatorname{div}_{x}\left(n_{\varepsilon}^{2} D_{y} \psi^{2}\left(\frac{x}{\varepsilon}\right)\right)+\frac{1}{\varepsilon} \nu^{2}\left(\frac{x}{\varepsilon}\right) n_{\varepsilon}^{2}=\frac{1}{\varepsilon} \nu^{1}\left(\frac{x}{\varepsilon}\right) n_{\varepsilon}^{1},
\end{array} \quad \text { in } \quad \mathbb{R}^{d} \times(0, \infty),\right.
$$

the only difference being in the value of the effective Hamiltonian $\bar{H}(\cdot)$-see Sect. 2. We also remark that we can also consider without any difficulty systems with more than two equations.

Our work is inspired from the ideas in wavefront propagation and large deviations $[5,17]$, the method of perturbed test functions in homogenization [16] and the methods used in the study of the concentration effects $[6,24]$ and motor effects [25-27].

Throughout the paper solutions are taken to be either classical, if smooth or, otherwise, in the viscosity sense. We refer to $[4,11]$ for a general introduction to the theory of the latter. In addition we denote by $C$ positive constants which are independent of $\varepsilon$ but may change from line to line. Moreover, $B_{r}(x)$ is the open ball in $\mathbb{R}^{d}$ centered at $x$ and of radius $r>0$ and $\bar{B}_{r}(x)$ stands for its closure. When $x=0$ we simply write $B_{r}$ and $\bar{B}_{r}$ respectively. Finally many statements in the paper hold for $i=1,2$ without any changes. Hence, unless necessary, we will not be repeating the "for $i=1,2$ ".

The paper is organized as follows. In Sect. 2 we introduce the cell problem corresponding to (9), we recall that it has a solution and we introduce $\bar{H}$. In Sect. 3 we present some preliminary facts about the family $\left(n_{\varepsilon}^{i}\right)_{\varepsilon>0}$ and study the properties of the families $\left(R_{\varepsilon}^{i}\right)_{\varepsilon>0}$ that are needed to prove the convergence to $\bar{R}^{i}, \underline{R}^{i}$. Theorem 1.2 is proved in Sect. 4 . Using the results on the asymptotic behavior of the family $\left(R_{\varepsilon}^{i}\right)_{\varepsilon>0}$ we prove Theorem 1.1 in Sect. 5 . The asymptotic behavior of the family $\left(n_{\varepsilon}^{i}\right)_{\varepsilon>0}$ in a more general setting is analyzed in Sect. 6. In Sect. 7 we compare our results with the case of the parabolic scaling in [15]. Finally, for the convenience of the reader, we present in the Appendix a sketch of the proof of the solvability of the cell problem and the properties of $\bar{H}$.

\section{The cell problem}

In view of the presence of the exponential terms in (9) it is natural to expect that the $R_{\varepsilon}^{1}$ 's and $R_{\varepsilon}^{2}$ 's converge, if at all, as $\varepsilon \rightarrow 0$ to the same limit $R$. Following [27] we insert in (9) the formal expansion

$$
R_{\varepsilon}^{i}(x, t)=R(x, t)+\varepsilon \phi^{i}\left(\frac{x}{\varepsilon}\right)+O^{i}\left(\varepsilon^{2}\right),
$$


and, keeping only the terms multiplying $\varepsilon^{0}$ and writing $y$ for the fast variable $\frac{x}{\varepsilon}$, we conclude that

$$
\left\{\begin{array}{rr}
R_{t}-\Delta_{y} \phi^{1}+\left|D_{y} \phi^{1}+D_{x} R\right|^{2}-D_{y} \psi \cdot\left(D_{y} \phi^{1}+D_{x} R\right) & \\
+\Delta_{y} \psi+\nu^{2} \exp \left(\phi^{1}-\phi^{2}\right)=\nu^{1} & \\
R_{t}-\Delta_{y} \phi^{2}+\left|D_{y} \phi^{2}+D_{x} R\right|^{2}+\nu^{1} \exp \left(\phi^{2}-\phi^{1}\right)=\nu^{2} . & \text { in } \mathbb{R}^{d} \times(0, \infty)
\end{array}\right.
$$

The goal is then to come up with $\phi^{i}$ 's so that (15) is independent of $y$. This leads to the problem to find, for each $p \in \mathbb{R}^{d}$, a unique constant $\bar{H}(p)$ such that the system, which is usually called the cell problem,

$$
\begin{cases}-\Delta_{y} \phi^{1}+\left|D_{y} \phi^{1}+p\right|^{2}-D_{y} \psi \cdot\left(D_{y} \phi^{1}+p\right)+\Delta_{y} \psi & \\ +\nu^{2} \exp \left(\phi^{1}-\phi^{2}\right)=\nu^{1}+\bar{H}(p) & \text { in } \mathbb{R}^{d}, \\ -\Delta_{y} \phi^{2}+\left|D_{y} \phi^{2}+p\right|^{2}+\nu^{1} \exp \left(\phi^{2}-\phi^{1}\right)=\nu^{2}+\bar{H}(p), & \end{cases}
$$

admits an 1-periodic solution $\left(\phi^{1}, \phi^{2}\right)$ called the corrector.

We have:

Lemma 2.1. For each $p \in \mathbb{R}^{d}$ there exists a unique constant $\bar{H}(p)$ such that (16) has an 1-periodic solution $\left(\phi^{1}, \phi^{2}\right)$. Moreover, $\bar{H} \in \mathrm{C}^{1}\left(\mathbb{R}^{d}\right), \bar{H}(0)=0, \bar{H}$ is strictly convex, there exists a constant $C$ such that $\bar{H}(p) \geq|p|^{2}-C$, and, hence, $\bar{H}(p) \rightarrow \infty$ as $|p| \rightarrow \infty$.

A proof of Lemma 2.1 for $d=1$ was included in [27]. For the convenience of the reader we sketch in the Appendix the proof in $\mathbb{R}^{d}$. by

When considering the system (14) with two potentials, (16) is replaced

$\left\{\begin{array}{l}-\Delta_{y} \phi^{1}+\left|D_{y} \phi^{1}+p\right|^{2}-D_{y} \psi^{1} \cdot\left(D_{y} \phi^{1}+p\right)+\Delta_{y} \psi^{1}+\nu^{2} \exp \left(\phi^{1}-\phi^{2}\right)=\nu^{1}+\bar{H}(p) \\ -\Delta_{y} \phi^{2}+\left|D_{y} \phi^{2}+p\right|^{2}-D_{y} \psi^{2} \cdot\left(D_{y} \phi^{2}+p\right)+\Delta_{y} \psi^{2}+\nu^{1} \exp \left(\phi^{2}-\phi^{1}\right)=\nu^{2}+\bar{H}(p),\end{array}\right.$ in $\mathbb{R}^{d}$.

\section{Some preliminaries and the properties of $\boldsymbol{R}_{\varepsilon}^{i}$}

We summarize in the next lemma some basic properties of the families $\left(n_{\varepsilon}^{i}\right)_{\varepsilon>0}$. They are the conservation of mass, the strict positivity of the $n_{\varepsilon}^{i}$ 's, and a global upper bound yielding that, as $\varepsilon \rightarrow 0$, there is very little mass at infinity.

We have:

Lemma 3.1. (i) For all $t \geq 0$ and $\varepsilon>0$,

$$
\int_{\mathbb{R}^{d}} n_{\varepsilon}^{1}(x, t) d x+\int_{\mathbb{R}^{d}} n_{\varepsilon}^{2}(x, t) d x=I_{\varepsilon}^{0} .
$$

(ii) Assume (2) and (3). Then

$$
0<n_{\varepsilon}^{i} \text { in } \mathbb{R}^{d} \times[0,+\infty) .
$$


(iii) Assume, in addition, (6). There exists $D>0$ such that, for all $(x, t) \in$ $\mathbb{R}^{d} \times[0,+\infty)$.

$$
n_{\varepsilon}^{i}(x, t) \leq \exp \left(\frac{-A|x|+B+D t}{\varepsilon}\right) .
$$

In particular, there is small mass at infinity, i.e., for all $t \geq 0$, there exists $M=M(t)>0$ such that

$$
\int_{|x| \geq M} n_{\varepsilon}^{i}(t, x) d x \underset{\varepsilon \rightarrow 0}{\longrightarrow} 0 .
$$

Proof. The conservation of the mass follows from adding the equations in (1) and integrating over $\mathbb{R}^{d}$.

The form of (1), (2) and (3) allow us to use maximum principle-type arguments to obtain (18) and (19).

Indeed let

$$
F_{1, \varepsilon}(n)=n_{t}-\varepsilon \Delta n-\operatorname{div}_{x}\left(n D_{y} \psi\left(\frac{x}{\varepsilon}\right)\right)+\frac{1}{\varepsilon} \nu^{1}\left(\frac{x}{\varepsilon}\right) n-\frac{1}{\varepsilon} \nu^{2}\left(\frac{x}{\varepsilon}\right) n,
$$

and

$$
F_{2, \varepsilon}(n)=n_{t}-\varepsilon \Delta n+\frac{1}{\varepsilon} \nu^{2}\left(\frac{x}{\varepsilon}\right) n-\frac{1}{\varepsilon} \nu^{1}\left(\frac{x}{\varepsilon}\right) n .
$$

It is easy to verify that $n_{\varepsilon}=\min \left(n_{\varepsilon}^{1}, n_{\varepsilon}^{2}\right)$ and $N_{\varepsilon}=\max \left(n_{\varepsilon}^{1}, n_{\varepsilon}^{2}\right)$ satisfy, in $\mathbb{R}^{d} \times(0, \infty)$, respectively

$$
\max \left(F_{1, \varepsilon}\left(n_{\varepsilon}\right), F_{2, \varepsilon}\left(n_{\varepsilon}\right)\right) \geq 0 \text { and } \min \left(F_{1, \varepsilon}\left(N_{\varepsilon}\right), F_{2, \varepsilon}\left(N_{\varepsilon}\right)\right) \leq 0 .
$$

Since 0 is clearly a solution of the first inequality in (21), (18) follows from the strong maximum principle.

To prove (19) we observe that, for $D$ sufficiently large,

$$
G_{\varepsilon}(x, t)=\exp \left(\frac{-A|x|+B+D t}{\varepsilon}\right)
$$

is a viscosity supersolution of the second inequality in (21). Since, in view of (6), we also have

$$
N_{\varepsilon}(x, 0) \leq G_{\varepsilon}(x, 0),
$$

we conclude using again the comparison principle.

Finally (20) follows from (19) after an appropriate choice of the constant $M$.

We turn now to the properties of the $R_{\varepsilon}^{i}$ 's which are presented in Theorem 3.2 below. The proof is rather long. The first part, which provides an one-sided Lipschitz-type continuity in time, is based on the classical Harnack inequality. The lower bound in part(ii) follows from part(i). The arguments leading to the upper bound (part(iii)) are more tedious and require as an intermediary step, namely, the construction, again using part(i), of an appropriate local upper bound. 
We have:

Theorem 3.2. (i) Assume (2) and (3). For all $\delta>0$, there exists $C_{\delta}>0$, such that, for all $\varepsilon>0,\left|z-z^{\prime}\right| \leq \varepsilon, \varepsilon \delta \leq t_{0}$ and $i, j=1,2$,

$$
R_{\varepsilon}^{j}\left(z^{\prime}, t_{0}+\varepsilon\right)-R_{\varepsilon}^{i}\left(z, t_{0}\right) \leq \varepsilon C_{\delta} .
$$

(ii) Assume (2), (3), (4) and (5). For any $a \in(0, \infty)$, there exists $\varepsilon_{0}=$ $\varepsilon_{0}(a)>0$ such that, for all $\varepsilon \leq \varepsilon_{0}$,

$$
R_{\varepsilon}^{i} \geq-a \text { in } \mathbb{R}^{d} \times[0, \infty) .
$$

(iii) Assume (2), (3), (4) and (6). For any compact subset $K$ of $\mathbb{R}^{d} \times$ $(0, \infty)$, there exist $C_{K}>0$ and $\varepsilon_{1}=\varepsilon_{1}(K)>0$ such that, for all $\varepsilon \leq \varepsilon_{1}$ and $(x, t) \in K$,

$$
R_{\varepsilon}^{i}(x, t) \leq C_{K}
$$

Proof. We begin with the

Proof of (22): Observe that $\widetilde{n}^{1}(y, \tau)=n_{\varepsilon}^{1}(\varepsilon y, \varepsilon \tau)$ and $\widetilde{n}^{2}(y, \tau)=n_{\varepsilon}^{2}(\varepsilon y, \varepsilon \tau)$ are positive (recall (18)) solutions to

$$
\left\{\begin{array}{l}
\tilde{n}_{\tau}^{1}-\Delta_{y} \widetilde{n}^{1}-\widetilde{n}^{1} \Delta_{y} \psi(y)-D_{y} \psi(y) \cdot D_{y} \widetilde{n}^{1}+\nu^{1} \widetilde{n}^{1}=\nu^{2} \widetilde{n}^{2} \\
\widetilde{n}_{\tau}^{2}-\Delta_{y} \widetilde{n}^{2}+\nu^{2} \widetilde{n}^{2}=\nu^{1} \widetilde{n}^{1},
\end{array} \quad \text { in } \mathbb{R}^{d} \times(0, \infty)\right.
$$

a linear parabolic system with bounded, according to (2), coefficients. It follows from the classical Harnack inequality [23] that, for each $\delta>0$, there exists, an independent of $\varepsilon, C_{\delta}>0$ such that for all $y_{0} \in \mathbb{R}^{d}, \tau_{0} \geq \delta$ and $i, j=1,2$,

$$
\sup _{z \in B_{1}\left(y_{0}\right)} \widetilde{n}^{i}\left(z, \tau_{0}\right) \leq C_{\delta} \inf _{z \in B_{1}\left(y_{0}\right)} \widetilde{n}^{j}\left(z, \tau_{0}+1\right) .
$$
we get

Rewriting (26) in terms of $n^{1}$ and $n^{2}$ and in the original variables $(x, t)$

$$
\sup _{z \in B_{\varepsilon}\left(x_{0}\right)} n_{\varepsilon}^{i}\left(z, t_{0}\right) \leq C_{\delta} \inf _{z \in B_{\varepsilon}\left(x_{0}\right)} n_{\varepsilon}^{j}\left(z, t_{0}+\varepsilon\right), \quad \text { for }\left(x_{0}, t_{0}\right) \in \mathbb{R}^{d} \times[\varepsilon \delta,+\infty) .
$$

Finally using (8) we obtain (22).

We continue with the

Proof of the uniform bounds from below: Arguing by contradiction we assume that for some $a>0$ there exist $\varepsilon_{k} \rightarrow 0$ and $\left(x_{k}, t_{k}\right) \in \mathbb{R}^{d} \times[0, \infty)$ such that

$$
\min \left(R_{\varepsilon_{k}}^{1}\left(x_{k}, t_{k}\right), R_{\varepsilon_{k}}^{2}\left(x_{k}, t_{k}\right)\right)<-a .
$$

Newt observe that, in view of (5) and (6), for $\varepsilon \leq \varepsilon_{a}$ with $\varepsilon_{a}$ small enough, we have

$$
\min \left(R_{\varepsilon_{k}}^{1}\left(x_{k}, t_{k}\right), R_{\varepsilon_{k}}^{2}\left(x_{k}, t_{k}\right)\right)>-\frac{a}{2}
$$


Moreover $\min \left(R_{\varepsilon}^{1}, R_{\varepsilon}^{2}\right)$ is a supersolution to

$$
\begin{gathered}
\max \left(R_{\varepsilon, t}^{1}-\varepsilon \Delta_{x} R_{\varepsilon}^{1}+\left|D_{x} R_{\varepsilon}^{1}\right|^{2}-D_{y} \psi\left(\frac{x}{\varepsilon}\right) \cdot D_{x} R_{\varepsilon}^{1}+\Delta_{y} \psi\left(\frac{x}{\varepsilon}\right)+\nu^{2}\left(\frac{x}{\varepsilon}\right)-\nu^{1}\left(\frac{x}{\varepsilon}\right),\right. \\
\left.R_{\varepsilon, t}^{2}-\varepsilon \Delta_{x} R_{\varepsilon}^{2}+\left|D_{x} R_{\varepsilon}^{2}\right|^{2}+\nu^{1}\left(\frac{x}{\varepsilon}\right)-\nu^{2}\left(\frac{x}{\varepsilon}\right)\right) \geq 0
\end{gathered}
$$

which admits $-\frac{a}{2}-c t$ as a subsolution provided $c$ is chosen sufficiently large.

It follows that, for $\varepsilon \leq \varepsilon_{a}$ and $t \geq 0$,

$$
\min \left(R_{\varepsilon}^{1}(\cdot, t), R_{\varepsilon}^{2}(\cdot, t)\right) \geq-\frac{a}{2}-c t, \quad \text { in } \mathbb{R}^{d},
$$

and therefore, if $\delta=\frac{a}{2 c}$, for $\varepsilon \leq \varepsilon_{a}$,

$$
\min \left(R_{\varepsilon}^{1}, R_{\varepsilon}^{2}\right) \geq-a, \quad \text { in } \mathbb{R}^{d} \times[0, \delta] .
$$

As a result the sequence $\left(x_{k}, t_{k}\right)$ chosen at the beginning of the proof must satisfy $t_{k}>\delta$.

Using (22) we deduce that there exists $C_{1}>0$ such that, as $k \rightarrow \infty$ and for all $x$ such that $\left|x-x_{k}\right| \leq \varepsilon_{k}$ and $i=1,2$,

$$
R_{\varepsilon_{k}}^{i}\left(x, t_{k}+\varepsilon_{k}\right) \leq R_{\varepsilon_{k}}^{i}\left(x_{k}, t_{k}\right)+C_{1} \varepsilon_{k} \leq-a+C_{1} \varepsilon_{k}
$$

It follows that

$$
\int_{\mathbb{R}^{d}} n_{\varepsilon_{k}}^{i}\left(x, t_{k}+\varepsilon_{k}\right) d x \geq \int_{\left|x-x_{k}\right| \leq \varepsilon_{k}} e^{-\frac{R_{\varepsilon_{k}}^{i}\left(x, t_{k}+\varepsilon_{k}\right)}{\varepsilon_{k}}} d x \geq\left|B_{\varepsilon_{k}}\left(x_{k}\right)\right| e^{\frac{a}{\varepsilon_{k}}-C_{1}} .
$$

The right hand side of this inequality blows up as $k \rightarrow \infty$, while the left hand side is bounded in view of (17) and (4), again a contradiction.

The last part of the proof is devoted to the

Proof of the uniform upper bounds on compact: Fix a compact subset $K$ of $\mathbb{R}^{d} \times(0, \infty)$, observe that

$$
t_{0}=\inf \left\{s \in(0, \infty): \text { there exists } x \in \mathbb{R}^{d} \text { such that }(x, s) \in K\right\}>0,
$$

choose $t_{1} \in\left(0, t_{0}\right)$ and write $\bar{t}_{1}=t_{1} / 2$ and $\bar{t}_{2}=t_{1} / 4$.

It follows from (4), (17) and (20) that there exist $\varepsilon_{1}>0$ and $M>0$ both dependent on $t_{1}$ such that, if $\varepsilon \leq \varepsilon_{1}$, then

$$
\int_{|x| \leq M} n_{\varepsilon}^{1}\left(x, \bar{t}_{1}\right)+n_{\varepsilon}^{2}\left(x, \bar{t}_{1}\right) d x \geq \frac{I_{0}}{2},
$$

and, hence, in view of (8), there exists some $a>0$ such that

$$
\min _{\substack{|x| \leq M \\ i=1,2}} R_{\varepsilon}^{i}\left(x, \bar{t}_{1}\right) \leq b=-\ln \left(\frac{a I_{0}}{M^{d}}\right) \text {. }
$$

Assume next that the above minimum is achieved at some point $x_{\varepsilon} \in \bar{B}_{M}$ and for $i=i^{*}$. Applying (22) $L=\left\lfloor\frac{t_{1}}{2 \varepsilon}\right\rfloor$ times with $i=i^{*}, j=2, x_{0}=x_{\varepsilon}$ and $\delta=\bar{t}_{1}$, we find some $C=C_{\delta}>0$ such that, for all $x \in B_{L \varepsilon}\left(x_{\varepsilon}\right)$,

$$
R_{\varepsilon}^{2}\left(x, \bar{t}_{1}+L \varepsilon\right) \leq b+C L \varepsilon \leq b+C \bar{t}_{1} .
$$


Choose $\gamma \geq b+C \bar{t}_{1}$ and for some $\beta>0$ to be fixed below define $\phi^{1}$ : $B_{\bar{t}_{1}}\left(x_{\varepsilon}\right) \times(0, \infty) \rightarrow \mathbb{R}$ by

$$
\phi^{1}(x, t)=\frac{1}{\bar{t}_{1}^{2}-\left|x-x_{\varepsilon}\right|^{2}}+\beta t+\gamma
$$

We claim that, for $\varepsilon \leq \varepsilon_{2}=\min \left(\bar{t}_{2}, \varepsilon_{1}\right)$,

$$
R_{\varepsilon}^{2} \leq \phi^{1} \quad \text { in } \quad Q_{\varepsilon}=B_{\bar{t}_{1}}\left(x_{\varepsilon}\right) \times\left[\bar{t}_{1}+L \varepsilon,+\infty\right),
$$

and, therefore,

$$
R_{\varepsilon}^{2} \leq \phi^{1} \quad \text { in } \quad Q_{\varepsilon}^{1}=B_{\bar{t}_{1}}\left(x_{\varepsilon}\right) \times\left[t_{1},+\infty\right) .
$$

To prove (29) we first notice that, in view of (28) and the choice of $\gamma$,

$$
R_{\varepsilon}^{2}\left(\cdot, \bar{t}_{1}+L \varepsilon\right) \leq b+C \bar{t}_{1} \leq \gamma \leq \phi^{1}\left(\cdot, \bar{t}_{1}+L \varepsilon\right) \quad \text { in } \quad B_{\mathbf{t}_{\mathbf{1}}}\left(x_{\varepsilon}\right)
$$

Moreover, if $\beta$ is large enough, using (2), for all $(x, t) \in Q_{\varepsilon}$, we have

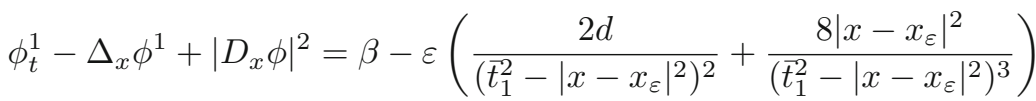

$$
\begin{aligned}
& +\frac{4\left|x-x_{\varepsilon}\right|^{2}}{\left(\bar{t}_{1}^{2}-\left|x-x_{\varepsilon}\right|^{2}\right)^{4}}>D=\max _{y \in \mathbb{R}^{d}} \nu^{2}(y) .
\end{aligned}
$$

The inequality above and (9) yield that, for all $(x, t) \in Q_{\varepsilon}$,

$$
\phi_{t}^{1}-\varepsilon \Delta_{x} \phi^{1}+\left|D_{x} \phi^{1}\right|^{2}+\nu^{1}\left(\frac{\dot{\varepsilon}}{\varepsilon}\right) \exp \left(\frac{R_{\varepsilon}^{2}-R_{\varepsilon}^{1}}{\varepsilon}\right)>\nu^{2} .
$$

Since $R_{\varepsilon}^{2} \leq \phi^{1}$ on the parabolic boundary of $Q_{\varepsilon}$, (29) follows from the maximum principle.

For $\varepsilon \leq \varepsilon_{2}$ let $Q^{2}=\left(\mathbb{R}^{d} \backslash B_{\bar{t}_{2}}\left(x_{\varepsilon}\right)\right) \times\left(t_{1},+\infty\right)$ and for positive constants $\alpha, \eta, \zeta$ to be fixed below consider the map $\phi^{2}:\left(\mathbb{R}^{d} \backslash B_{\bar{t}_{2}}\left(x_{\varepsilon}\right)\right) \times\left(t_{1},+\infty\right) \rightarrow \mathbb{R}$ given by

$$
\phi^{2}(x, t)=\frac{\alpha\left|x-x_{\varepsilon}\right|^{2}}{t-t_{1}}+\eta t+\zeta .
$$

We claim that

$$
R_{\varepsilon}^{2} \leq \phi^{2} \quad \text { in } \quad Q^{2} .
$$

As above we will show that, if we choose $\alpha, \eta$ and $\zeta$ appropriately, $\phi^{2}$ is a supersolution in $Q^{2}$ of the equation satisfied by $R_{\varepsilon}^{2}$ and is above $R_{\varepsilon}^{2}$ on the parabolic boundary of $Q^{2}$.

To this end notice that, in view of (30), we may select $\zeta$ and $\eta$ large enough so that, for $\varepsilon \leq \varepsilon_{2}$,

$$
R_{\varepsilon}^{2} \leq \eta t+\zeta \leq \phi^{2} \quad \text { on } \quad \partial B_{\bar{t}_{2}}\left(x_{\varepsilon}\right) \times\left(t_{1}, \infty\right) .
$$

Moreover, for sufficiently large $\alpha, \eta$, we have

$$
\phi_{t}^{1}-\Delta_{x} \phi^{1}+\left|D_{x} \phi\right|^{2}=\eta-\frac{\alpha\left|x-x_{\varepsilon}\right|^{2}}{\left(t-t_{1}\right)^{2}}-\frac{2 \varepsilon \alpha d}{t-t_{1}}+\frac{4 \alpha^{2}\left|x-x_{\varepsilon}\right|^{2}}{\left(t-t_{1}\right)^{2}}>D,
$$


and, hence, in $Q^{2}$,

$$
\phi_{t}^{2}-\varepsilon \Delta_{x} \phi^{2}+\left|D_{x} \phi^{2}\right|^{2}+\nu^{1}\left(\frac{\cdot}{\varepsilon}\right) \exp \left(\frac{R_{\varepsilon}^{2}-R_{\varepsilon}^{1}}{\varepsilon}\right)>\nu^{2}\left(\frac{\cdot}{\varepsilon}\right) .
$$

Since clearly $R_{\varepsilon}^{2}\left(\cdot, t_{1}\right) \leq \phi^{2}\left(\cdot, t_{1}\right)$ in $\left(\mathbb{R}^{d} \backslash B_{\bar{t}_{2}}\left(x_{\varepsilon}\right)\right)$, using again the maximum principle we find (31).

To conclude observe that (30) and (31) yield that the family $\left(R_{\varepsilon}^{2}\right)_{\varepsilon>0}$ is uniformly bounded from above in any compact subset of $\mathbb{R}^{d} \times\left(t_{1}, \infty[\right.$ and thus, in particular, on $K$, for $\varepsilon \leq \varepsilon_{1}$. Finally, using again (22), we deduce that the family $\left(R_{\varepsilon}^{1}\right)_{\varepsilon>0}$ is also uniformly bounded from above on $K$, for $\varepsilon \leq$ $\min \left(\varepsilon_{1}, t_{0}-t_{1}\right)$.

\section{Convergence to the Homogenized equation-The proof of Theorem 1.2}

Before we begin the proof, we remark that, in view of the claimed properties of $\bar{H}$ (convexity and coercivity), the equation

$$
R_{t}+\bar{H}\left(R_{x}\right)=0 \text { in } \mathbb{R}^{d} \times(0, \infty),
$$

admits a comparison principle even for initial data taking the value $\infty$ (see [12]). However, this and Theorem 1.2 do not lead to $\bar{R} \leq \underline{R}$. Indeed we show in the next section that, in addition to (12) in Theorem 1.2, the conservation of mass yields

$$
\underline{R}(0,0)=\underline{R}^{1}(0,0)=\underline{R}^{2}(0,0)=0 .
$$

To be able to use the comparison principle it is necessary to have $\bar{R}^{1}(0,0)=$ $\bar{R}^{2}(0,0) \leq 0$, which is not possible. Indeed, since $\bar{R}^{1}$ and $\bar{R}^{2}$ are upper semicontinuous, (12) implies $\bar{R}^{1}(0,0)=\bar{R}^{2}(0,0)=+\infty$.

We continue with the

Proof of Theorem 1.2. It is immediate from (22) that (i) holds. Moreover it follows from Theorem 3.2 (ii) that

$$
0 \leq \underline{R}^{1} \leq \bar{R}^{1} \text { and } 0 \leq \underline{R}^{2} \leq \bar{R}^{2} \text { on } \mathbb{R}^{d} \times[0, \infty),
$$

and thus, in particular, the second part of (12).

To prove (11) we employ the so called perturbed test function method. Since the arguments are similar here we only show that $\bar{R}$ is a subsolution of (11).

To this end we assume that, for some smooth $\varphi, \bar{R}-\varphi$ achieves a strict local maximum at $\left(x_{0}, t_{0}\right) \in \mathbb{R}^{d} \times(0, \infty)$. Following [27], we perturb $\varphi$ using the solution $\left(\phi^{1}, \phi^{2}\right)$ of the cell problem (16) corresponding to $p=D \varphi\left(x_{0}, t_{0}\right)$. It follows that there exists $\left(x_{\varepsilon}, t_{\varepsilon}\right) \in \mathbb{R}^{d} \times(0, \infty) \rightarrow\left(x_{0}, t_{0}\right)$, as $\varepsilon \rightarrow 0$, such that the $\max _{i=1,2}\left(R_{\varepsilon}^{i}-\varphi-\varepsilon \phi^{i}(\dot{\bar{\varepsilon}})\right)$ is attained at $\left(x_{\varepsilon}, t_{\varepsilon}\right)$ and, without any loss of generality since the argument is identical, for $i=1$. Hence,

$$
R_{\varepsilon}^{1}\left(x_{\varepsilon}, t_{\varepsilon}\right)-\varepsilon \phi^{1}\left(\frac{x_{\varepsilon}}{\varepsilon}\right) \geq R_{\varepsilon}^{2}\left(x_{\varepsilon}, t_{\varepsilon}\right)-\varepsilon \phi^{2}\left(\frac{x_{\varepsilon}}{\varepsilon}\right) .
$$


That $R_{\varepsilon}^{1}$ is a solution of (9) yields subsolution test we obtain

$$
\begin{aligned}
& \varphi_{t}\left(x_{\varepsilon}, t_{\varepsilon}\right)-\varepsilon \Delta_{x} \varphi\left(x_{\varepsilon}, t_{\varepsilon}\right)-\Delta_{y} \phi^{1}\left(\frac{x_{\varepsilon}}{\varepsilon}\right)+\left|D_{x} \varphi\left(x_{\varepsilon}, t_{\varepsilon}\right)+D_{y} \phi^{1}\left(\frac{x_{\varepsilon}}{\varepsilon}\right)\right|^{2} \\
& -D_{y} \psi\left(\frac{x_{\varepsilon}}{\varepsilon}\right) \cdot\left(D_{x} \varphi\left(x_{\varepsilon}, t_{\varepsilon}\right)+D_{x} \phi^{1}\left(\frac{x_{\varepsilon}}{\varepsilon}\right)\right)+\Delta_{y} \psi\left(\frac{x_{\varepsilon}}{\varepsilon}\right) \\
& +\nu^{2}\left(\frac{x_{\varepsilon}}{\varepsilon}\right) \exp \left(\frac{R_{\varepsilon}^{1}-R_{\varepsilon}^{2}}{\varepsilon}\right) \leq \nu^{1}\left(\frac{x_{\varepsilon}}{\varepsilon}\right) .
\end{aligned}
$$

Using the latter and (34) and writing $p_{\varepsilon}=D \varphi\left(x_{\varepsilon}, t_{\varepsilon}\right)$ we get

$$
\begin{aligned}
& \varphi_{t}\left(x_{\varepsilon}, t_{\varepsilon}\right)-\varepsilon \Delta_{x} \varphi\left(x_{\varepsilon}, t_{\varepsilon}\right)-\Delta_{y} \phi^{1}\left(\frac{x_{\varepsilon}}{\varepsilon}\right)+\left|p_{\varepsilon}+D_{y} \phi^{1}\left(\frac{x_{\varepsilon}}{\varepsilon}\right)\right|^{2} \\
& -D_{y} \psi\left(\frac{x_{\varepsilon}}{\varepsilon}\right) \cdot\left(p_{\varepsilon}+D_{x} \phi^{1}\left(\frac{x_{\varepsilon}}{\varepsilon}\right)\right)+\Delta_{y} \psi\left(\frac{x_{\varepsilon}}{\varepsilon}\right) \\
& +\nu^{2}\left(\frac{x_{\varepsilon}}{\varepsilon}\right) \exp \left(\phi^{1}\left(\frac{x_{\varepsilon}}{\varepsilon}\right)-\phi^{2}\left(\frac{x_{\varepsilon}}{\varepsilon}\right)\right) \leq \nu^{1}\left(\frac{x_{\varepsilon}}{\varepsilon}\right) .
\end{aligned}
$$

It follows from the above inequality and the definition of the effective Hamiltonian (16) that, for some $o(1) \rightarrow 0$ as $\varepsilon \rightarrow 0$,

$$
\varphi_{t}\left(x_{\varepsilon}, t_{\varepsilon}\right)-\varepsilon \Delta_{x} \varphi\left(x_{\varepsilon}, t_{\varepsilon}\right)+\bar{H}\left(D_{x} \varphi\left(x_{\varepsilon}, t_{\varepsilon}\right)\right) \leq o(1),
$$

and the conclusion follows letting $\varepsilon \rightarrow 0$.

To prove the first part of (12), since the arguments are identical, here we only show that $\underline{R}^{1}(\cdot, 0)=\bar{R}^{1}(\cdot, 0)=\infty$ in $\mathbb{R}^{d} \backslash\{0\}$. To this aim, we fix $\kappa>0$, we select an auxiliary function $\xi \in C^{\infty}\left(\mathbb{R}^{d}\right)$ such that $0<\xi(x)<1$ for $x \in$ $\mathbb{R}^{d} \backslash\{0\}$ and $\xi(0)=0$, and prove that

$$
\max \left(\underline{R}_{t}^{1}+\bar{H}\left(\underline{R}_{x}^{1}\right), \underline{R}^{i}-\kappa \xi\right) \geq 0 \quad \text { in } \mathbb{R}^{d} \times[0, \infty) .
$$

Since we already know that $(35)$ holds in $\mathbb{R}^{d} \times(0, \infty)$, to conclude we assume that, for a smooth $\phi, \underline{R}^{1}-\phi$ achieves a (strict) local maximum in $\left(x_{0}, 0\right)$ and we prove that either

$$
\underline{R}^{1}\left(x_{0}, 0\right) \geq \kappa \xi\left(x_{0}\right),
$$

or

$$
\phi_{t}\left(x_{0}, 0\right)+\bar{H}\left(\phi_{x}\left(x_{0}, 0\right)\right) \geq 0 .
$$

If $x_{0}=0$, the former is clearly true since $\underline{R}^{1}(0,0) \geq 0=\kappa \xi(0)$.

So we assume that $\underline{R}^{1}-\phi$ has a local maximum in $\left(x_{0}, 0\right)$ with $x_{0} \neq 0$ and, in addition, that $\underline{R}^{1}\left(x_{0}, 0\right)<\kappa \xi\left(x_{0}\right)$. Repeating the arguments used earlier in the proof we obtain, for some $\left(x_{\varepsilon}, t_{\varepsilon}\right) \rightarrow\left(x_{0}, 0\right)$ as $\varepsilon \rightarrow 0$, we have

$$
\varphi_{t}\left(x_{\varepsilon}, t_{\varepsilon}\right)-\varepsilon \Delta_{x} \varphi\left(x_{\varepsilon}, t_{\varepsilon}\right)+\bar{H}\left(D_{x} \varphi\left(x_{\varepsilon}, t_{\varepsilon}\right)\right) \geq o(1) .
$$

Indeed (5) and the facts that $\underline{R}^{1}\left(x_{0}, 0\right)<\kappa \xi\left(x_{0}\right)<\kappa$ and $\lim _{\varepsilon \rightarrow 0} R_{\varepsilon}^{1}(y, 0)=$ $+\infty$ for all $y$ near $x_{0}$ yield that $t_{\varepsilon}>0$. The claim now follows by letting $\varepsilon \rightarrow 0$.

Assume next that, for some $x_{0} \neq 0, \underline{R}^{1}\left(x_{0}, 0\right)=b<+\infty$. We fix $\delta>0$ and let

$$
\mu^{\delta}(x, t)=-\frac{\left|x-x_{0}\right|^{2}}{\delta}-\gamma t
$$

for $\gamma=\gamma(\delta)>0$ to be determined later. 
Since $\underline{R}^{1}$ is lower semicontinuous, $\underline{R}^{1}-\mu^{\delta}$ attains a minimum at some $\left(x_{\delta}, t_{\delta}\right) \in \mathbb{R}^{d} \times[0, \infty)$ such that, as $\delta \rightarrow 0,\left(x_{\delta}, t_{\delta}\right) \rightarrow\left(x_{0}, 0\right)$, provided that $\gamma \rightarrow \infty$ as $\delta \rightarrow 0$.

Observe next that the choice of $\left(x_{\delta}, t_{\delta}\right)$ yields

$$
\frac{\left|x_{\delta}-x_{0}\right|^{2}}{\delta} \leq \underline{R}^{1}\left(x_{\delta}, t_{\delta}\right)+\frac{\left|x_{\delta}-x_{0}\right|^{2}}{\delta}+\gamma t_{\delta} \leq \underline{R}^{1}\left(x_{0}, 0\right)=b,
$$

and, hence,

$$
\left|x_{\delta}-x_{0}\right| \leq \sqrt{b \delta} .
$$

If $t_{\delta}>0$, according to part (ii), we must have $\mu_{t}^{\delta}\left(x_{\delta}, t_{\delta}\right)+\bar{H}\left(D \mu^{\delta}\left(x_{\delta}, t_{\delta}\right)\right)$ $\geq 0$ and, hence,

$$
-\gamma+\bar{H}\left(-\frac{2\left(x_{\delta}-x_{0}\right)}{\delta}\right) \geq 0
$$

which cannot be true if we choose $\gamma>\sup _{|x| \leq \sqrt{b \delta}} \bar{H}\left(-\frac{2 x}{\delta}\right)$.

Now we assume that $t_{\delta}=0$. If $\underline{R}^{1}\left(x_{0}, 0\right)<\kappa \xi\left(x_{0}\right)$, then (37) yields $\underline{R}^{1}\left(x_{\delta}, 0\right)<\kappa_{\delta} \xi\left(x_{\delta}\right)$ for some $\kappa_{\delta} \rightarrow \kappa$ as $\delta \rightarrow 0$. Using (35) with $\kappa_{\delta}$ at the point $\left(x_{\delta}, 0\right)$ we obtain again (38) and thus a contradiction.

It follows that $\underline{R}^{1}\left(x_{0}, 0\right)>\kappa \xi\left(x_{0}\right)$, which also leads to a contradiction, since it holds for arbitrarily large $\kappa$ and $\underline{R}^{1}\left(x_{0}, 0\right)=b<+\infty$.

The first part of (12) now follows.

\section{The transport of the concentration points: the proof of Theorem 1.1}

We present here the

Proof. (Proof of Theorem 1.1) Using (11), (12), the standard optimal control formula $[3,18,22]$ and a barrier argument similar to the one of Section 5 in [17], we obtain that $\underline{R}$ satisfies

$$
\underline{R}(x, t) \geq \inf _{\substack{\zeta(s), s) \in \mathbb{R}^{d} \times(0, \infty)+\\ \zeta(0)=0, \zeta(t)=x}} \int_{0}^{t} H^{\star}(\dot{\zeta}(s)) d s+\max \left(\underline{R}^{1}(0,0), \underline{R}^{2}(0,0)\right),
$$

with $H^{\star}(p)=\sup _{q \in \mathbb{R}^{d}}(p \cdot q-\bar{H}(q))$.

Observe next that, since Lemma 2.2 yields

$$
\lim _{|q| \rightarrow+\infty} p \cdot q-\bar{H}(q) \geq \lim _{|q| \rightarrow+\infty} p \cdot q-|q|^{2}+C=-\infty,
$$

the maximum of $(p \cdot q-\bar{H}(q))$ is attained at some $q_{p} \in \mathbb{R}^{d}$ such that $p=$ $D \bar{H}\left(q_{p}\right)$.

Moreover, since $\bar{H}$ is strictly convex and $\bar{H}(0)=0$, we have

$$
D \bar{H}\left(q_{p}\right) \cdot q_{p}-\bar{H}\left(q_{p}\right) \geq 0 \text { with equality only if } q_{p}=0 .
$$

Hence we deduce that

$$
H^{*}(p)>0 \text { for all } p \neq D \bar{H}(0) \text { and } H^{*}(D \bar{H}(0))=0 .
$$


Therefore, using (39) and (12), we obtain that

$$
\begin{aligned}
& \underline{R} \geq 0 \text { in } \mathbb{R}^{d} \times \mathbb{R}^{+} \text {and if } \underline{R}(x, t)=0, \text { then } x=t D \bar{H}(0) \\
& \text { and } \underline{R}^{1}(0,0)=\underline{R}^{2}(0,0)=0 .
\end{aligned}
$$

It follows from the latter, (8) and (10) that, for $i=1,2$,

$$
\limsup _{\substack{(y, s) \rightarrow(x, t) \\ \varepsilon \rightarrow 0}} n_{\varepsilon}^{i}(y, s)=0 \text { in } \mathbb{R}^{d} \times[0,+\infty) \backslash\{(t D \bar{H}(0), t) \mid t \in[0,+\infty)\} .
$$

Finally the last claim and (17) yield that the $\left(n_{\varepsilon}^{1}+n_{\varepsilon}^{2}\right)$ 's converge weakly along subsequences to a measure $n$ with

$$
\operatorname{supp} n \subset\{(t \nabla \bar{H}(0), t) \mid t \in[0, \infty)\} .
$$

Since we also know that, according to (20), no mass escapes to infinity as $\varepsilon \rightarrow 0$, we deduce (7) using (17). Moreover, in view of (40), we obtain that

$$
\underline{R}^{1}(0,0)=\underline{R}^{2}(0,0)=0 .
$$

\section{The case with several Dirac masses initially}

We proved Theorem 1.1 under assumptions (5) which imply that the densities $n_{\varepsilon}^{1}$ and $n_{\varepsilon}^{2}$ are both initially concentrated at the origin. The result can be generalized to densities concentrated at several points and probably not on the same points. If this is the case, the initial condition is written as

$$
\left\{\begin{array}{l}
\lim _{\varepsilon \rightarrow 0} \varepsilon \ln n_{\varepsilon}^{1}(\text { cot, } 0)=\lim _{\varepsilon \rightarrow 0} \varepsilon \ln n_{\varepsilon}^{2}(\cdot, 0) \\
=-\infty \text { locally uniformly in } \mathbb{R}^{d} \backslash(\mathcal{A} \cup \mathcal{B}), \\
\text { and } \\
\limsup _{\substack{y \rightarrow x \\
\varepsilon \rightarrow 0}} \varepsilon \ln n_{\varepsilon}^{i}(y, 0) \leq 0 \quad \text { for all } x \in \mathcal{A} \cup \mathcal{B} .
\end{array}\right.
$$

We have:

Theorem 6.1. Assume (2), (3), (4), (6) and (41). Then, for $i=1,2$ and as $\varepsilon \rightarrow 0$, along subsequences and in the sense of measures, $n_{\varepsilon}^{1} \rightarrow n^{1}$ and $n_{\varepsilon}^{2} \rightarrow n^{2}$ with

$$
\begin{aligned}
& \operatorname{supp}\left(n^{1}+n^{2}\right)(\cdot, t) \subset \mathcal{C}(t)=\left\{x_{1}+t D \bar{H}(0), \ldots, x_{n}+t D \bar{H}(0)\right\} \cup \\
& \left\{y_{1}+t D \bar{H}(0), \ldots, y_{m}+t D \bar{H}(0)\right\} .
\end{aligned}
$$

Proof. The proof of Theorem 6.1 follows along the same lines as the one of Theorem 1.1. The only difference is that (12) and (39) are replaced respectively by

$$
\begin{gathered}
\bar{R}^{1}=\bar{R}^{2}=\underline{R}^{1}=\underline{R}^{2}=+\infty \text { in }\left(\mathbb{R}^{d} \backslash \mathcal{C}(0)\right) \times\{0\}, \\
\underline{R}^{1} \geq 0 \text { and } \underline{R}^{2} \geq 0 \text { in } \mathcal{C}(0) \times\{0\}
\end{gathered}
$$


and

$$
\underline{R}(x, t) \geq \inf _{\substack{\zeta(s), s) \in \mathbb{R}^{d} \times(0, \infty) \\ \zeta(0) \in \mathcal{C}(0), \zeta(t)=x}}\left\{\int_{0}^{t} H^{\star}(\dot{\zeta}(s)) d s+\max \left(\underline{R}^{1}(\zeta(0), 0), \underline{R}^{2}(\zeta(0), 0)\right)\right\} .
$$

Therefore, we have

$\underline{R} \geq 0$ in $\mathbb{R}^{d} \times(0, \infty)$ and if $\underline{R}(x, t)=0$, then $x \in \mathcal{C}(t)$.

The other parts of the proof are similar.

\section{A comparison with results for parabolic scaling}

We describe here the connection between our result and the study in [15] of the asymptotics, as $\varepsilon \rightarrow 0$, of the solutions to

$$
\begin{aligned}
n_{\varepsilon, t}=( & \left.a_{i j}\left(\frac{x}{\varepsilon}, \frac{t}{\varepsilon^{2}}\right) n_{\varepsilon, x_{j}}\right)_{x_{i}}+\frac{1}{\varepsilon} b_{i}\left(\frac{x}{\varepsilon}, \frac{t}{\varepsilon^{2}}\right) n_{\varepsilon, x_{i}} \\
& +\frac{1}{\varepsilon^{2}} c\left(\frac{x}{\varepsilon}, \frac{t}{\varepsilon^{2}}\right) n_{\varepsilon} \text { in } \mathbb{R}^{d} \times(0, \infty) .
\end{aligned}
$$

Notice that this is a single equation - not a system - obtained after a parabolic scaling $(x, t) \mapsto\left(\frac{x}{\varepsilon}, \frac{t}{\varepsilon^{2}}\right)$ rather than the hyperbolic scaling $(x, t) \mapsto$ $\left(\frac{x}{\varepsilon}, \frac{t}{\varepsilon}\right)$ of the problem we consider in this paper. Nevertheless, as we explain below, there are some similarities.

It is proved in [15] that the solution $n_{\varepsilon}$ of (43) admits the expansion

$$
n_{\varepsilon}(x, t)=w\left(\frac{x}{\varepsilon}, \frac{t}{\varepsilon^{2}}\right) \exp \left(-\frac{\lambda_{0} t}{\varepsilon^{2}}\right) v^{0}\left(x-\frac{\bar{b}}{\varepsilon} t, t\right)+o(1) .
$$

The result we obtain here with the hyperbolic scaling formally gives

$$
n_{\varepsilon}^{i}(x, t)=w^{i}\left(\frac{x}{\varepsilon}, \frac{t}{\varepsilon}\right) \rho^{i}(t) \delta(x-D \bar{H}(0) t)+o(1) .
$$

In particular we obtain a Dirac mass instead of the function $v^{0}$ while the term $\exp \left(-\frac{\lambda_{0} t}{\varepsilon^{2}}\right)$ disappears because we have conservation of mass. Moreover $t / \varepsilon$ is replaced by $t$, because of the difference in the scaling. There is, however, as we explain below a close connection between the function $w$ in (44) and $w^{i}$ in (45).

Indeed $w$ in [15] is the principal eigenvector of the periodic cell problem

$$
w_{s}-\left(a_{i j}(z, s) w_{z_{j}}\right)_{z_{i}}-b_{i}(z, s) w_{z_{i}}-c(z, s) w=\Lambda_{0} w,
$$

while we have

$$
w^{i}=\exp \left(-\phi^{i}\right)
$$

where $\left(\phi^{1}, \phi^{2}\right)$ is the principle eigenvector for the cell problem (16) corresponding to $p=0$ with the corresponding eigenvalue $\bar{H}(0)=0$. This is because for $(\bar{x}, \bar{t})$ in the support of $n^{i}$, we have $R(\bar{x}, \bar{t})=0$ and thus $D R(\bar{x}, \bar{t})=0$. 


\section{Acknowledgments}

This work has partly been done during a visit of the first author to the University of Chicago while supported by Foundation Sciences Mathématique de Paris. S. M. would like to thank the University of Chicago for its hospitality.

\section{Appendix A. The proof of Lemma 2.1}

Step 1: We prove that, for all $p \in \mathbb{R}^{d}$, there exists a unique $\bar{H}(p)$ with the properties stated in Lemma 2.1. Following [27], for $i=1,2$, we define

$$
\chi^{i}(y)=\exp \left(-p \cdot y-\phi^{i}(y)\right) .
$$

Multiplying the two equations in (16) by $\chi^{1}$ and $\chi^{2}$ respectively, we obtain

$$
\left\{\begin{array}{l}
-\Delta_{y} \chi^{1}-\operatorname{div}_{y}\left(D_{y} \psi \chi^{1}\right)+\nu^{1} \chi^{1}-\nu^{2} \chi^{2}=-\bar{H}(p) \chi^{1}, \\
-\Delta_{y} \chi^{2}+\nu^{2} \chi^{2}-\nu^{1} \chi^{1}=-\bar{H}(p) \chi^{2} .
\end{array}\right.
$$

with the boundary condition

$$
y \rightarrow e^{p . y} \chi^{i}(y) \text { is one-periodic and } \chi^{i}>0 .
$$

We also impose the normalization

$$
\int_{0}^{1}\left(\chi^{1}(y)+\chi^{2}(y)\right) d y=1 .
$$

It follows from the Krein-Rutman Theorem that, for all $p \in \mathbb{R}^{d}$, there exists a unique constant $\bar{H}(p)$ and functions $\left(\chi^{1}, \chi^{2}\right)$ satisfying (46) together with (47) and (48).

Step 2: To prove that $\bar{H} \in \mathrm{C}^{1}\left(\mathbb{R}^{d}\right)$ we rewrite (46) in terms of $w_{p}^{i}(y)=$ $e^{p \cdot y} \chi^{i}(y)$, which in view of $(47)$ are 1 - periodic, and obtain the new system

$$
\left\{\begin{array}{c}
-\Delta_{y} w_{p}^{1}+2 p \cdot D_{y} w_{p}^{1}-\operatorname{div}_{y}\left(D_{y} \psi w_{p}^{1}\right)+\left(-|p|^{2}+p \cdot D_{y} \psi+\nu^{1}\right) \\
w_{p}^{1}-\nu^{2} w_{p}^{2}=-\bar{H}(p) w_{p}^{1} \\
-\Delta_{y} w_{p}^{2}+2 p \cdot D_{y} w_{p}^{2}+\left(-|p|^{2}+\nu^{2}\right) w_{p}^{2}-\nu^{1} w_{p}^{1}=-\bar{H}(p) w_{p}^{2} .
\end{array}\right.
$$

Assuming for the moment that $w_{p}^{1}$ and $w_{p}^{2}$ are differentiable with respect to $p$, after differentiating (49) we get

$$
\left\{\begin{array}{l}
-\Delta_{y} \partial_{p} w_{p}^{1}+2 p \cdot D_{y} \partial_{p} w_{p}^{1}-\operatorname{div}_{y}\left(D_{y} \psi \partial_{p} w_{p}^{1}\right)+\left(-|p|^{2}+p \cdot \nabla_{y} \psi+\nu^{1}\right) \\
\quad \partial_{p} w_{p}^{1}-\nu^{2} \partial_{p} w_{p}^{2}+2 D_{y} w_{p}^{1}+\left(-2 p+D_{y} \psi\right) w_{p}^{1}=-\bar{H}(p) \partial_{p} w_{p}^{1}-\bar{H}^{\prime}(p) w_{p}^{1}, \\
-\Delta_{y} \partial_{p} w_{p}^{2}+2 p \cdot D_{y} \partial_{p} w_{p}^{2}+\left(-|p|^{2}+\nu^{2}\right) \partial_{p} w_{p}^{2}-\nu^{1} \partial_{p} w_{p}^{1}+2 D_{y} w_{p}^{2}-2 p w_{p}^{2} \\
\quad=-\bar{H}(p) \partial_{p} w_{p}^{2}-\bar{H}^{\prime}(p) w_{p}^{2} .
\end{array}\right.
$$

Let $\left(w_{p, *}^{1}, w_{p, *}^{2}\right)$ be the solution to the adjoint system of $(49)$ - note that Fredholm's alternative implies the existence of such a solution. Multiplying the 
equations of (49) by $w_{p, *}^{1}$ and $w_{p, *}^{2}$, integrating with respect to $y$ and adding the two resulting equations we obtain

$$
\begin{gathered}
2 \int w_{p, *}^{1} D_{y} w_{p}^{1} d y+2 \int w_{p, *}^{2} D_{y} w_{p}^{2} d y+\int D_{y} \psi w_{p}^{1} w_{p, *}^{1} d y \\
=(2 p-D \bar{H}(p))\left(\int w_{p}^{1} w_{p, *}^{1} d y+\int w_{p}^{2} w_{p, *}^{2} d y\right),
\end{gathered}
$$

which yields a formula for $D \bar{H}(p)$.

To prove the above claim rigorously, we write the system satisfied by difference quotients with respect to $p$ and we use the same idea as above to prove that the difference quotients $h^{-1}\left(\bar{H}\left(p+h e_{k}\right)-\bar{H}(p)\right)$ converge to the above formula, as $h \rightarrow 0$. Indeed from this formulation we first obtain that $\bar{H}(p)$ is continuous with respect to $p$. Then we show that $w_{p}^{1}$ and $w_{p}^{2}$ are continuous with respect to $p$ and, finally, we pass to the limit $h \rightarrow 0$.

To prove that $w_{p}^{1}$ and $w_{p}^{2}$ are continuous with respect to $p$, we show that, for any sequence $\left(p_{n}\right)_{n \in \mathbb{N}}$ such that $p_{n} \rightarrow p$, the corresponding eigenfunctions $\left(w_{p_{n}}^{1}, w_{p_{n}}^{2}\right)$ converge to $\left(w_{p}^{1}, w_{p}^{2}\right)$ as $n \rightarrow \infty$. This follows, for example, from the stability of viscosity solutions, the continuity of $\bar{H}$, the uniqueness (up to a multiplicative constant) of the eigenfunctions $\left(w_{p}^{1}, w_{p}^{2}\right)$ and the normalization condition (48).

Step 3: The proof of $\bar{H}(0)=0$.

The adjoint system to (46) is

$$
\left\{\begin{array}{l}
-\Delta_{y} u^{1}+D_{y} \psi \cdot D_{y} u^{1}+\nu^{1} u^{1}=\nu^{1} u^{2}-\bar{H}(p) u^{1}, \\
-\Delta_{y} u^{2}+\nu^{2} u^{2}=\nu^{2} u^{1}-\bar{H}(p) u^{2}
\end{array}\right.
$$

with the condition

$$
y \rightarrow e^{-p \cdot y} u^{i}(y) 1 \text {-periodic and } u^{i}>0 .
$$

When $p=0$, (50) admits the trivial solution $\left(u^{1}, u^{2}\right)=(1,1)$ and the constant $\bar{H}(0)=0$. The claim follows from the uniqueness of the positive eigenvector.

Step 4: The proof of the strict convexity of $\bar{H}(p)$.

Arguing by contradiction we assume that there exist $p_{1}, p_{2}$ such that

$$
\frac{\bar{H}\left(p_{1}\right)+\bar{H}\left(p_{2}\right)}{2} \leq \bar{H}\left(\frac{p_{1}+p_{2}}{2}\right) .
$$

Let $\left(\phi_{1}^{1}, \phi_{1}^{2}\right)$ and $\left(\phi_{2}^{1}, \phi_{2}^{2}\right)$ be solutions of (16) corresponding to $p=p_{1}$ and $p=p_{2}$ respectively. Adding the corresponding equations we find

$$
\begin{aligned}
-\Delta_{y} & \left(\frac{\phi_{1}^{1}+\phi_{2}^{1}}{2}\right)+\left|D_{y}\left(\frac{\phi_{1}^{1}+\phi_{2}^{1}}{2}\right)+\frac{p_{1}+p_{2}}{2}\right|^{2}-D_{y} \psi \cdot\left(D_{y}\left(\frac{\phi_{1}^{1}+\phi_{2}^{1}}{2}\right)+\frac{p_{1}+p_{2}}{2}\right)+\Delta_{y} \psi \\
& +\nu^{2} \exp \left(\frac{\phi_{1}^{1}+\phi_{2}^{1}-\phi_{1}^{2}-\phi_{2}^{2}}{2}\right) \\
< & -\Delta_{y}\left(\frac{\phi_{1}^{1}+\phi_{2}^{1}}{2}\right)+\frac{1}{2}\left|D_{y} \phi_{1}^{1}+p_{1}\right|^{2}+\frac{1}{2}\left|D_{y} \phi_{2}^{1}+p_{2}\right|^{2}-D_{y} \psi \cdot\left(\frac{D_{y} \phi_{1}^{1}+p_{1}}{2}\right) \\
& -D_{y} \psi \cdot\left(\frac{D_{y} \phi_{2}^{1}+p_{2}}{2}\right)+\Delta_{y} \psi+\frac{\nu^{2}}{2} \exp \left(\phi_{1}^{1}-\phi_{1}^{2}\right)+\frac{\nu^{2}}{2} \exp \left(\phi_{2}^{1}-\phi_{2}^{2}\right) \\
= & \left.\nu^{1}+\frac{\bar{H}\left(p_{1}\right)+\bar{H}\left(p_{2}\right)}{2}\right) \leq \nu^{1}+\bar{H}\left(\frac{p_{1}+p_{2}}{2}\right),
\end{aligned}
$$


and

$$
\begin{aligned}
- & \Delta_{y}\left(\frac{\phi_{1}^{2}+\phi_{2}^{2}}{2}\right)+\left|D_{y}\left(\frac{\phi_{1}^{2}+\phi_{2}^{2}}{2}\right)+\frac{p_{1}+p_{2}}{2}\right|^{2}+\nu^{1} \exp \left(\frac{\phi_{1}^{2}+\phi_{2}^{2}-\phi_{1}^{1}-\phi_{2}^{1}}{2}\right) \\
& <\nu^{2}+\bar{H}\left(\frac{p_{1}+p_{2}}{2}\right) .
\end{aligned}
$$

It follows that the pair $\left(\frac{\phi_{1}^{1}+\phi_{2}^{1}}{2}, \frac{\phi_{1}^{2}+\phi_{2}^{2}}{2}\right)$ is a strict subsolution to the cell problem (16) corresponding to $p=\frac{p_{1}+p_{2}}{2}$. This, however, contradicts the fact that $\bar{H}\left(\frac{p_{1}+p_{2}}{2}\right)$ is the principal eigenvalue of the system.

Step 5: The proof of $\bar{H}(p) \geq|p|^{2}-C$.

Rewriting (16) we find

$$
\left\{\begin{array}{l}
-\Delta_{y} \phi^{1}+\left|D_{y} \phi^{1}\right|^{2}-\left(D_{y} \psi-2 p\right) \cdot D_{y} \phi^{1}-D_{y} \psi \cdot p+\Delta_{y} \psi+\nu^{2} \exp \left(\phi^{1}-\phi^{2}\right) \\
=\nu^{1}+\bar{H}(p)-|p|^{2} \\
-\Delta_{y} \phi^{2}+\left|D_{y} \phi^{2}\right|^{2}+2 p \cdot D_{y} \phi^{2}+\nu^{1} \exp \left(\phi^{2}-\phi^{1}\right)=\nu^{2}+\bar{H}(p)-|p|^{2}
\end{array}\right.
$$

Assume next that $\max _{[0,1]}\left(\phi^{1}, \phi^{2}\right)$ is attained at the point $\bar{y}$. If $\phi^{1}(\bar{y})>$ $\phi^{2}(\bar{y})$, then

$$
-\Delta \phi^{1}(\bar{y}) \geq 0 \text { and } D \phi^{1}(\bar{y})=0
$$

and, hence,

$$
\bar{H}(p) \geq-\|\Delta \psi\|_{L^{\infty}}-|p|\left\|\left.D_{y} \psi\right|_{L^{\infty}}-\right\| \nu^{1} \|_{L^{\infty}}+|p|^{2} .
$$

If $\phi^{1}(\bar{y})>\phi^{2}(\bar{y})$, then, similarly, we find

$$
\bar{H}(p) \geq-\left\|\nu^{1}\right\|_{L^{\infty}}+|p|^{2} .
$$

The claim now follows.

\section{References}

[1] Allaire, G., Capdeboscq, Y.: Homogenization of a spectral problem in neutronic multigroup diffusion. Comput. Methods Appl. Mech. Eng. 187, 91-117 (2000)

[2] Astumian R.D, Hänggi, P.: Brownian motors. Phys. Today 55, 33-39 (2002)

[3] Bardi, M., Capuzzo-Dolcetta, I.: Optimal control and viscosity solutions of Hamilton-Jacobi-Bellman equations. In: Systems \& Control: Foundations \& Applications. With appendices by Maurizio Falcone and Pierpaolo Soravia. Birkhäuser Boston Inc., Boston (1997)

[4] Barles, G.: Solutions de viscosité des équations de Hamilton-Jacobi. Mathématiques \& Applications (Berlin) [Mathematics \& Applications], vol. 17. Springer, Paris (1994)

[5] Barles, G., Evans, L.C., Souganidis, P.E.: Wavefront propagation for reactiondiffusion systems of PDE. Duke Math. J. 61, 835-858 (1990)

[6] Barles, G., Perthame, B.: Concentrations and constrained Hamilton-Jacobi equations arising in adaptive dynamics. Contemp. Math. 439, 57-68 (2007) 
[7] Bena, I., Vanden Broeck, C., Copelli, M.: Stokes' drift: a rocking ratchet. J. Stat. Phys. 101, 415-423 (2000)

[8] Blanchet, A., Dolbeault, J., Kowalczyk, M.: Stochastic stokes drift, homogenized functional inequalities, and large time behaviour of brownian ratchets. SIAM J. Math. Anal. 41, 46-76 (2009)

[9] Chipot, M., Hastings, S., Kinderlehrer, D.: Transport in a molecular motor system, M2AN. Math. Model. Numer. Anal. 38, 1011-1034 (2004)

[10] Collet, P., Martinez, S.: Asymptotic velocity of one dimensional diffusions with periodic drift. J. Math. Biol. 56, 765-792 (2008)

[11] Crandall, M.G., Ishii, H., Lions, P.-L.: User's guide to viscosity solutions of second order partial differential equations. Bull. Am. Math. Soc. (N.S.) 27, $1-67(1992)$

[12] Crandall, M.G., Lions, P.-L., Souganidis, P.E.: Maximal solutions and universal bounds for some partial differential equations of evolution. Arch. Ration. Mech. Anal. 105, 163-190 (1989)

[13] Doering, C., Ermentrout, B., Oster, G.: Rotary DNA motors. Biophys. J. 69, 2256-2267 (1995)

[14] Dolbeault, J., Kinderlehrer, D., Kowalczyk, M.: Remarks about the flashing rachet. In: Partial Differential Equations and Inverse Problems. Contemp. Math., vol. 362, pp. 167-175. Amer. Math. Soc., Providence (2004)

[15] Donato, P., Piatnitski, A.: Averaging of nonstationary parabolic operators with large lower order terms. In: Multiscale Problems and Asymptotic Analysis. GAKUTO Int. Ser. Math. Sci. Appl., vol. 24, pp. 153-166. Gokkotosho, Tokyo (2005)

[16] Evans, L.: The perturbed test function method for viscosity solutions of nonlinear PDE. Proc. R. Soc. Edinb. Sec. A 111, 359-375 (1989)

[17] Evans, L.C., Souganidis, P.E.: A PDE approach to certain large deviation problems for systems of parabolic equations. Ann. Inst. H. Poincare Anal. Non Lineaire 6, 229-258 (1989)

[18] Fleming, W.H., Soner, H.M.: Controlled Markov processes and viscosity solutions. In: Stochastic Modelling and Applied Probability, vol. 25, 2nd edn. Springer, New York (2006)

[19] Huxley, A.F.: Muscle structure and theories of contraction. Prog. Biophys. Chem. 7, 255-318 (1957)

[20] Jülicher, F., Ajdari, A., Prost, J.: Modeling molecular motors. Rev. Modern Phys. 69, 1269-1281 (1997)

[21] Kinderlehrer, D., Kowalczyk, M.: Diffusion-mediated transport and the flashing ratchet. Arch. Ration. Mech. Anal. 161, 149-179 (2002) 
[22] Lions, P.L.: Generalized solutions of Hamilton-Jacobi equations. Research Notes in Mathematics, vol. 69. Pitman Advanced Publishing Program, Boston (1982)

[23] Moser, J.: A harnack inequality for parabolic differential equations. Commun. Pure Appl. Math. 17, 101-134 (1964)

[24] Perthame, B., Barles, G.: Dirac concentrations in Lotka-Volterra parabolic PDEs. Indiana Univ. Math. J. 57, 3275-3301 (2008)

[25] Perthame, B., Souganidis, P.E.: Asymmetric potentials and motor effect: a large deviation approach. Arch. Ration. Mech. Anal. 193, 153-169 (2009)

[26] Perthame, B., Souganidis, P.E.: A homogenization approach to flashing ratchets. Nonlinear Differ. Equ. Appl. 18, 45-58 (2011)

[27] Perthame, B., Souganidis, P.E.: Asymmetric potentials and motor effect: a homogenization approach. Annales de l'Institut Henri Poincare (C) Non Linear Analysis 26, 2055-2071 (2009)

S. Mirrahimi

CMAP, Ecole Polytechnique, CNRS, INRIA

Route de Saclay, 91128 Palaiseau Cedex, France

e-mail: mirrahimi@cmap.polytechnique.fr

P. E. Souganidis

Department of Mathematics

The University of Chicago

5734 S. University Avenue, Chicago, IL 60637, USA

e-mail: souganidis@math.uchicago.edu

Received: 16 December 2011.

Accepted: 7 February 2012. 